\title{
Consistency of recommendations for the diagnosis and treatment of non-small cell lung cancer: a systematic review
}

\author{
Zhe Zhang ${ }^{1}$, Sen Yang ${ }^{1}$, Yanfang $\mathrm{Ma}^{2}$, Hanqiong Zhou ${ }^{1}$, Xuan $\mathrm{Wu}^{1}$, Jing $\mathrm{Han}^{1}$, Jiabao Hou ${ }^{1}$, Lidan Hao ${ }^{1}$, \\ Jonathan D. Spicer ${ }^{3}$, Young Wha Koh ${ }^{4}$, Mariano Provencio ${ }^{5}$, Noemi Reguart ${ }^{6}$, Tetsuya Mitsudomi ${ }^{7}$, \\ Qiming Wang ${ }^{1}$
}

${ }^{1}$ Department of Internal Medicine, The Affiliated Cancer Hospital of Zhengzhou University, Henan Cancer Hospital, Zhengzhou, China; ${ }^{2}$ School of Chinese Medicine of Hong Kong Baptist University, Kowloon Tong, Hong Kong, China; ${ }^{3}$ Division of Thoracic and Upper Gastrointestinal Surgery, Department of Surgery, McGill University Health Centre, McGill University, Montreal, QC, Canada; ${ }^{4}$ Department of Pathology, Ajou University School of Medicine, Suwon, Republic of Korea; ${ }^{5}$ Medical Oncology Department, Hospital Universitario Puerta de Hierro Majadahonda, Majadahonda, Madrid, Spain; ${ }^{6}$ Thoracic Oncology Unit, Department of Medical Oncology, IDIPAPS, Hospital Clinic Barcelona, Villarroel, Spain; ${ }^{7}$ Division of Thoracic Surgery, Department of Surgery, Kindai University Faculty of Medicine, Osaka, Japan

Contributions: (I) Conception and design: Q Wang, Y Ma, Z Zhang, S Yang; (II) Administrative support: Q Wang; (III) Provision of study materials or patients: Z Zhang, Y Ma, H Zhou, X Wu, J Han; (IV) Collection and assembly of data: Z Zhang, H Zhou, X Wu, J Han, J Hou, L Hao; (V) Data analysis and interpretation: Z Zhang, S Yang, Y Ma, H Zhou, X Wu; (VI) Manuscript writing: All authors; (VII) Final approval of manuscript: All authors.

Correspondence to: Qiming Wang, MD, PhD. Department of Internal Medicine, Henan Cancer Hospital Affiliated to Zhengzhou University, 127 Dong Ming Road, Zhengzhou 450008, China. Email: qimingwang1006@126.com.

Background: To systematically assess the consistency of recommendations regarding diagnosis and treatment of non-small cell lung cancer (NSCLC) in clinical practice guidelines (CPGs).

Methods: We systematically searched relevant literature databases and websites to identify CPGs related to NSCLC. We extracted the general characteristics of the included guidelines and their recommendations and descriptively compared and analyzed the consistency of recommendations across the guidelines.

Results: A total of 28 NSCLC guidelines were retrieved. The recommendations covered mainly diagnosis and treatment. The recommendations in the guidelines differed substantially in various topics, such as the application of positron emission tomography (PET) and the classification of stage III. Fourteen guidelines divided stage III into two types: operable and inoperable; and the remaining 14 guidelines into three sub-stages IIIA, IIIB and IIIC. Recommendations regarding the treatment in stage III were relatively inconsistent. In driver gene (EGFR, ALK, ROS1) positive patients, targeted therapy was the most common recommendation for first-line treatment, but recommendations regarding second-line treatment varied according to the site of the mutation. In driver gene negative patients, immunotherapy was the most frequently recommended option as both first- and second-line treatment, followed by chemotherapy.

Discussion: A number of countries are devoting themselves to develop NSCLC guidelines and the process of updating guidelines is accelerating, yet recommendations between guidelines are not consistent. We adopted a systematic review method to systematically search and analyze the NSCLC guidelines worldwide. We objectively reviewed the differences in recommendations for NSCLC diagnosis and treatment between the guidelines. Inconsistency of recommendations across guidelines can result from multiple potential reasons. Such as, the guidelines developed time, different countries and regions and many more. Poor consistency across CPGs can confuse the guideline users, and we therefore advocate paying more attention to examining the controversies and updating guidelines timely to improve the consistency among CPGs. Our study had also several limitations, we limited the search to CPGs published in Chinese or English, the interpretation of recommendations is inherently subjective, we did not evaluate the details of the clinical content of the CPG recommendations. Our research presents the current status of NSCLC guidelines worldwide and give the opportunity to pay more attention to the existing gaps. Further investigations should 
determine the reasons for inconsistency, the implications for recommendation development, and the role of synthesis across recommendations for optimal guidance of clinical care treatment. With the continuous revision and update of the guidelines, we are confident that future guidelines will be formulated with higher quality to form clear, definite and consistent recommendations for NSCLC diagnosis and treatment.

Keywords: Non-small cell lung cancer (NSCLC); clinical practice guidelines (CPGs); recommendations; consistency

Submitted Mar 16, 2021. Accepted for publication Jun 04, 2021.

doi: $10.21037 /$ tlcr-21-423

View this article at: http://dx.doi.org/10.21037/tlcr-21-423

\section{Introduction}

Lung cancer is the most common type of cancer type worldwide in terms of both incidence (2.1 million new cases in 2018) and mortality (1.8 million deaths in 2018). The key cause of lung cancer is tobacco smoking, which is responsible for $63 \%$ of overall global deaths from lung cancer and for more than $90 \%$ of lung cancer deaths in countries where smoking is prevalent among both men and women (1). The morbidity of lung cancer is however declining: the annual reduction in incidence grew from $3 \%$ between 2008 and 2013 to 5\% between 2013 through 2017 in men, and from $2 \%$ to almost $4 \%$ in women, spurring the largest ever single-year drop in overall cancer mortality of $2.2 \%$ from 2016 to 2017. Yet lung cancer still caused more deaths in 2017 than breast, prostate, colorectal, and brain cancers combined (2). Almost one-quarter of all cancer deaths are due to lung cancer $(2,3)$. Non-small cell lung cancer (NSCLC) is the most common type of lung cancer, accounting for about $80 \%$ of all lung cancers. Screening of high-risk population groups, early diagnosis, individualized treatment, targeted therapy and immunotherapy have contributed to the increase in 5 -year survival probability from $17.2 \%$ in 2009 to $21.7 \%$ in $2019(4,5)$. However, due to different treatment options, this progress differs across countries and regions. Active promotion of the optimal treatment pathways for NSCLC will therefore improve clinical outcomes.

Clinical practice guidelines (CPGs) provide clinical practitioners a basis for decision-making, and play an important role in standardizing behavior in medical care, improving patient prognosis, and saving medical resources $(6,7)$. In recent years, an increasing amount of CPGs for NSCLC have been published. However, there are differences in the classifications and terms used to describe the level of evidence and the strength of the recommendations between different guidelines, which can lead to confusion and complicate the choice of the most suitable guidelines to guide clinical work (8). Conversely, guidelines must represent the diversity and inequities in access to modern care occurring at a global level and provide support as to how to manage these issues. As well, important differences in prevalence of various NSCLC patient scenarios that occur within specific geographic areas can reasonably account for regional peculiarities of specific CPGs.

This study systematically retrieved the CPGs for NSCLC and analyzed the content of the guidelines in order to provide references for the diagnosis and treatment of NSCLC, and suggestions for the formulation of related guidelines in the future. We present the following article in accordance with the PRISMA reporting checklist (available at http://dx.doi.org/10.21037/tlcr-21-423).

\section{Methods}

\section{Search strategy}

We searched Medline (via PubMed), Chinese Biomedical Literature database (CBM), WanFang Database, China National Knowledge Infrastructure (CNKI), as well as the websites of Guidelines International Network (GIN), National Institute for Health and Clinical Excellence (NICE), World Health Organisation (WHO), Scottish Intercollegiate Guidelines Network (SIGN), and National Comprehensive Cancer Network (NCCN). A manual search in Google Scholar was also performed to find relevant NSCLC CPGs outside the databases. We used the following search terms: lung neoplasm, lung cancer, lung, guideline, guidance and recommendation. The search was restricted to the time period January 1, 2018 to November 15, 2020. The full search strategy is presented in Supplementary file (Appendix 1). 


\section{Inclusion and exclusion criteria}

We included guidelines for diagnosis and treatment of NSCLC published in either Chinese or English. If we found two or more versions of guidelines issued by the same organization and on the same topic, we only included the latest version.

We excluded introductions, analyses and applications of CPGs, and translations of guidelines.

\section{Literature screening}

Two investigators independently screened the titles and abstracts of the records identified in the initial search, and then the full texts of the potentially relevant articles. After the screening was completed, the results were compared. In case of disagreement, a third researcher was invited to discuss and resolve the inclusion of the article.

\section{Data extraction}

Two investigators independently carried out data extraction using a pre-designed information extraction table, followed by cross-checking. Differences were resolved by discussion. We extracted the following basic characteristics: title, year of publication, developer agency, country of the main developer, quality evaluation/grading system used, and the number of recommendations. We also extracted information on whether there were recommendations for all commonly used diagnostic methods and treatment plans, and the system used for grading the evidence.

The progression of lung cancer staging has relied on a TNM staging system, developed by the International Association for the Study of Lung Cancer (IASLC) $(9,10)$. NSCLC is divided into four stages (I-IV), and each stage has specific treatment strategies (10-12). We summarized and compared the recommendations on diagnostic methods (pathological diagnosis, molecular pathological diagnosis, imaging diagnosis) and treatment methods for the different stages of NSCLC.

\section{Data analysis}

We performed a descriptive analysis of the recommendations for diagnostic and treatment methods in the CPGs. We reported the number of recommendations in each field and the number of guidelines reporting specific recommendations.

\section{Results}

\section{Basic information}

A total of 657 related documents were initially retrieved, of which 651 were from literature databases and six from websites of organizations and associations. After excluding duplicate documents, reading titles and abstracts, and reading the full texts, we finally included 28 guidelines addressing diagnosis and treatment of NSCLC, five in Chinese and 23 in English (Figure 1). The guidelines came from ten countries or regions. Guideline development processes were variable. Seventeen $(60.7 \%)$ guidelines presented the recommendations in a way that allows to quantify their number. The number of recommendations in these guidelines ranged from 9 to 161 (mean: 47; median: 26). Clinical questions were specified in 7 (25\%) guidelines. Twenty-three guidelines $(82.1 \%)$ rated the quality of evidence and/or the strength of recommendations, among which $6(21.4 \%)$ used the GRADE classification system. Table 1 lists the characteristics of the included guidelines.

The content of the recommendations covered diagnosis and treatment of stage I, stage II, stage III and stage IV NSCLC, including pathological diagnosis, molecular diagnosis, imaging diagnosis, surgical treatment, targeted therapy, and immunotherapy.

\section{Consistency of recommendations}

The guidelines covered three types of diagnosis: pathological diagnosis (Figure 2), molecular diagnosis (Figure 3), and imaging diagnosis (Figure 4). For most recommendations their strength was not declared. There were a total of 83 recommendations for molecular diagnostics. Molecular diagnosis recommendations are given for specific gene sites, of which EGFR ( $\mathrm{n}=11$, $39.2 \%)$, ALK ( $n=11,39.2 \%)$ and ROS1 ( $n=11,39.2 \%)$ were mentioned by highest number of CPGs. Recommendations regarding imaging diagnosis were relatively inconsistent across the guidelines. The tests that were most commonly recommended were positron emission tomography (PETCT; $\mathrm{n}=17,60.7 \%)$, chest computed tomography (CT; $\mathrm{n}=16$, $57.1 \%)$ and ultrasound bronchoscopy ( $\mathrm{n}=16,57.1 \%)$.

Recommendations about which treatment to use varied by NSCLC stage and driving gene status (Tables 2-6). Six guidelines recommended multidisciplinary consultations for the treatment of stage III patients; and one guideline only recommended multidisciplinary consultations for stage IIIA patients (38). Different guidelines used different 


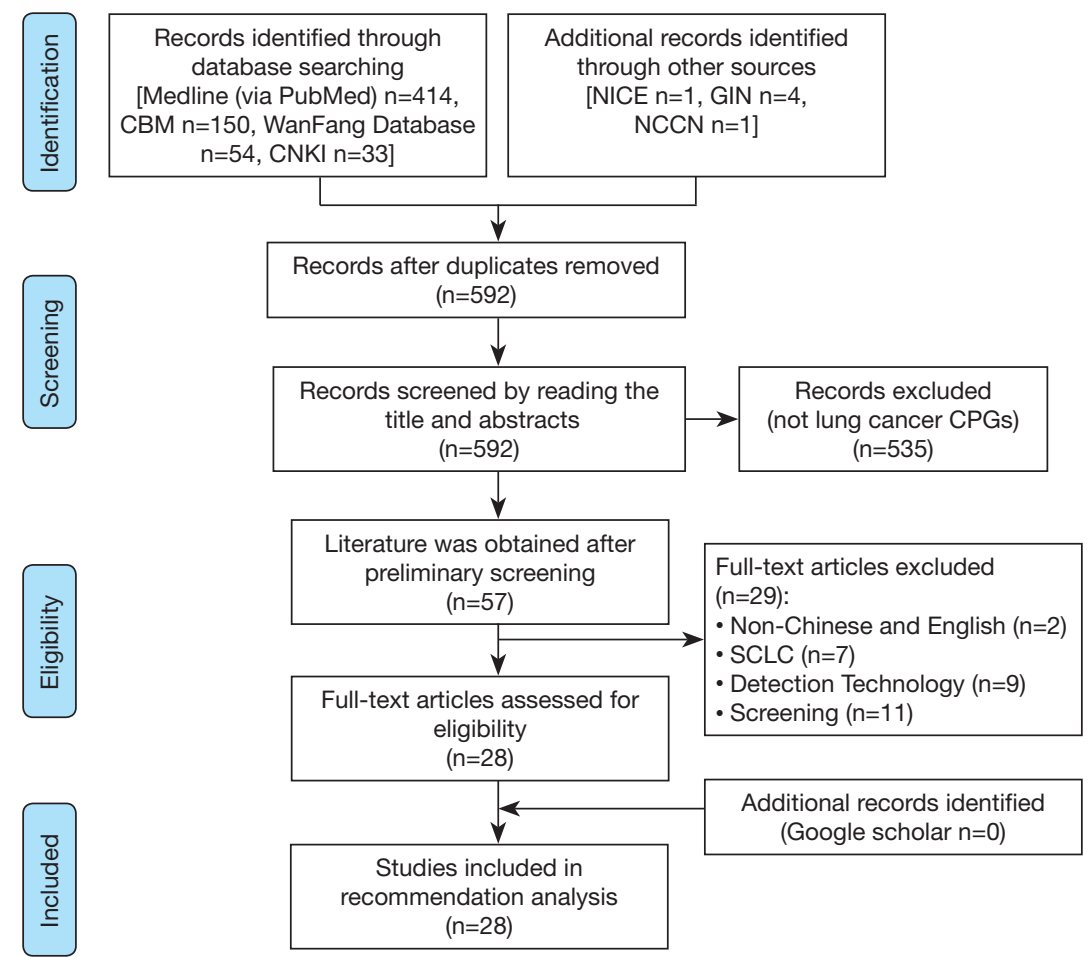

Figure 1 Flow diagram of the search and selection of the guidelines. CBM, Chinese Biomedical Literature database; CNKI, China National Knowledge Infrastructure; NICE, National Institute for Health and Clinical Excellence; GIN, Guidelines International Network; NCCN, National Comprehensive Cancer Network; CPGs, Clinical practice guidelines; SCLC, small cell lung cancer.

sub-classifications of stage III. Fourteen guidelines divided III into two types: operable and inoperable (Table 4); the remaining 14 guidelines divided stage III into three subtypes (stage IIIA, stage IIIB and stage IIIC; Table 5). Recommendations regarding the treatment of stage III were relatively inconsistent.

In stage IV, for EGFR-mutation positive patients, 15 (53.6\%) guidelines recommended targeted therapy as a part of first-line treatment. and 12 (42.9\%) guidelines as a part of second-line treatment. Five guidelines pointed out that targeted therapy should be used in second-line treatment in the event of a T790M mutation. In ALKrearrangement positive patients, targeted therapy was also commonly recommended for first-line treatment $(\mathrm{n}=14$, $50.0 \%)$ and second line treatment $(\mathrm{n}=12,42.9 \%)$. In ROS1rearrangement positive patients, targeted therapy was also recommended by $14(50.0 \%)$ guidelines for first-line treatment, whereas for second-line treatment, chemotherapy $(\mathrm{n}=8,28.6 \%)$ was the most common recommendation.

In driver gene negative patients, immunotherapy was the most frequently recommended treatment for both first- and second-line treatment, followed by chemotherapy. Only three clinical guidelines had recommendations for thirdline treatment (Table 6).

\section{Discussion}

In this study, we identified 28 NSCLC CPGs by a systematic literature search, most of which were produced nationally. Most but not all guidelines used and described a formal method to grade the quality of evidence. Recommendations on the diagnosis of NSCLC varied across guidelines. In clinical practice, pathological diagnosis is the gold standard for tumor diagnosis (41). Traditionally, tissue-based histopathological approaches play a major role in the diagnostics of lung cancer. We found that more than half of the guidelines did not give any recommendations on histomorphological or immunohistochemical diagnosis. This could be due to the slow progress in research on this topic, as the main focus of the diagnosis methods has been on molecular pathology (41). The importance of molecular pathology has become even more important with 







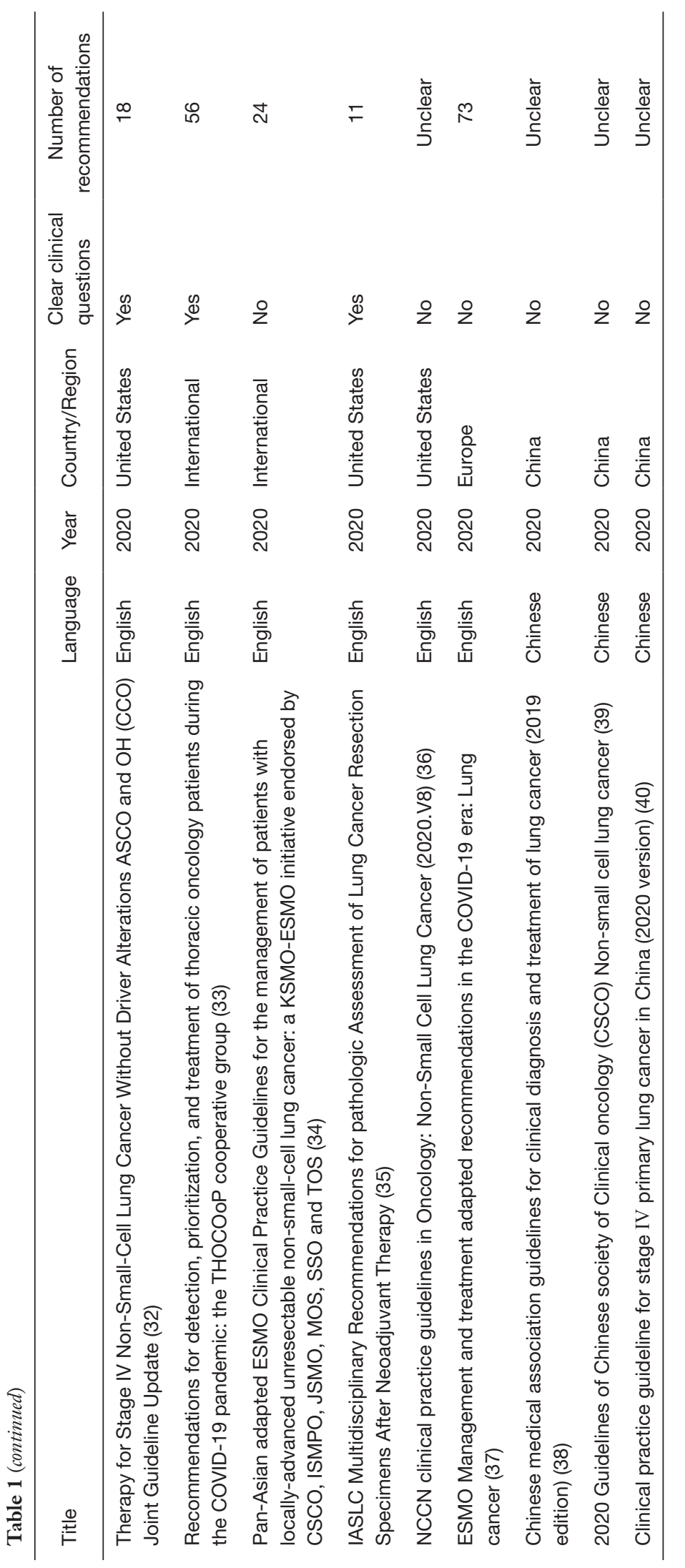






Figure 2 Recommendations for pathological diagnosis in the included clinical practice guidelines (unspecified strength: the evidence is not graded in the guidelines, or the evidence is graded but the strength of recommendations is not stated; not mentioned: the recommendation is not mentioned in the guideline).



Figure 3 Recommendations for molecular diagnosis recommendations in the included clinical practice guidelines (unspecified strength: the evidence is not graded in the guidelines, or the evidence is graded but the strength of recommendations is not stated; not mentioned: the recommendation is not mentioned in the guideline).

the introduction of individualized treatment options. The recommendations we found focused on the genes EGFR, ALK, ROS1, BRAF V600E and PD-L1 (42), because these driver genes have a high frequency of mutations, and because sufficient clinical trial data and relevant highquality evidence exists.

Targeted therapy is one of the main treatment options for NSCLC, especially in the first- and second-line clinical treatment of driver gene positive patients in advanced stage of the cancer. An increasing number of targeted drugs are entering the market and becoming relatively easy to obtain for clinicians and patients (43-47). Immunotherapy has significantly altered the treatment landscape for many types of cancer, including NSCLC. Currently approved immunooncology agents for lung cancer are aimed at the reversal of immune checkpoints, PD-1 and PD-L1 (48). The elevated expression of PD-L1 has been shown to correlate with higher efficacy of various immunotherapeutic agents, implying a high predictive value of this biomarker (49).

The most commonly recommended test for imaging 




Figure 4 Recommendations for imaging diagnosis in the included clinical practice guidelines (unspecified strength: the evidence is not graded in the guidelines, or the evidence is graded but the strength of recommendations is not stated; not mentioned: the recommendation is not mentioned in the guideline).

Table 2 Recommendations in the clinical practice guidelines for the treatment of stage I non-small cell lung cancer

\begin{tabular}{|c|c|c|c|c|}
\hline Stage & Treatment method & \multicolumn{3}{|c|}{ Number of clinical practice guidelines recommending each treatment method } \\
\hline \multicolumn{5}{|l|}{ Stage IA } \\
\hline Operable & Radical surgery & 3 & 8 & 17 \\
\hline Inoperable & Radical radiotherapy & 3 & 9 & 16 \\
\hline Operable & Radical radiotherapy & 3 & 7 & 18 \\
\hline Inoperable & Radical radiotherapy & 3 & 9 & 16 \\
\hline High-risk & Adjuvant chemotherapy & 0 & 3 & 25 \\
\hline
\end{tabular}

a, unspecified strength: the evidence was not graded in the guidelines, or the evidence was graded but the strength of the recommendation was not stated.

diagnosis of NSCLC was PET-CT. Metabolic parameters including standardized uptake value, metabolic tumor volume and total lesion glycolysis measured by PET-CT are associated with tumor aggressiveness and can provide additional prognostic information. However, it is difficult to use this method in economically underdeveloped areas due to the high price and lack of equipment $(50,51)$.

The treatment of NSCLC is based on staging $(27,33)$. For patients in stages I and II, radical surgery with or without adjuvant chemotherapy is recommended; for inoperable patients, radical radiotherapy with or without adjuvant chemotherapy is the most suitable choice. Stage III lung cancer is a medium-term lung cancer. If the patient undergoes standardized and precise treatment, NSCLC is still possible to be "clinically cured" in this stage. Stage III lung cancer is divided into two types (operable and inoperable), but the boundary between the two is not standardized because there is no unified staging and treatment plan for patients with mediastinal lymph node metastasis $(37,46,47,52-55)$. Regardless of the staging method, the treatment recommendations for Stage III were based on surgery, combined with different treatment methods such as chemotherapy and radiotherapy. In addition, some guidelines recommended radical concurrent chemoradiation as the first-line option for stage III operable patients, which is an extrapolation that has no basis in high 
Table 3 Recommendations in the clinical practice guidelines for the treatment of stage II non-small cell lung cancer

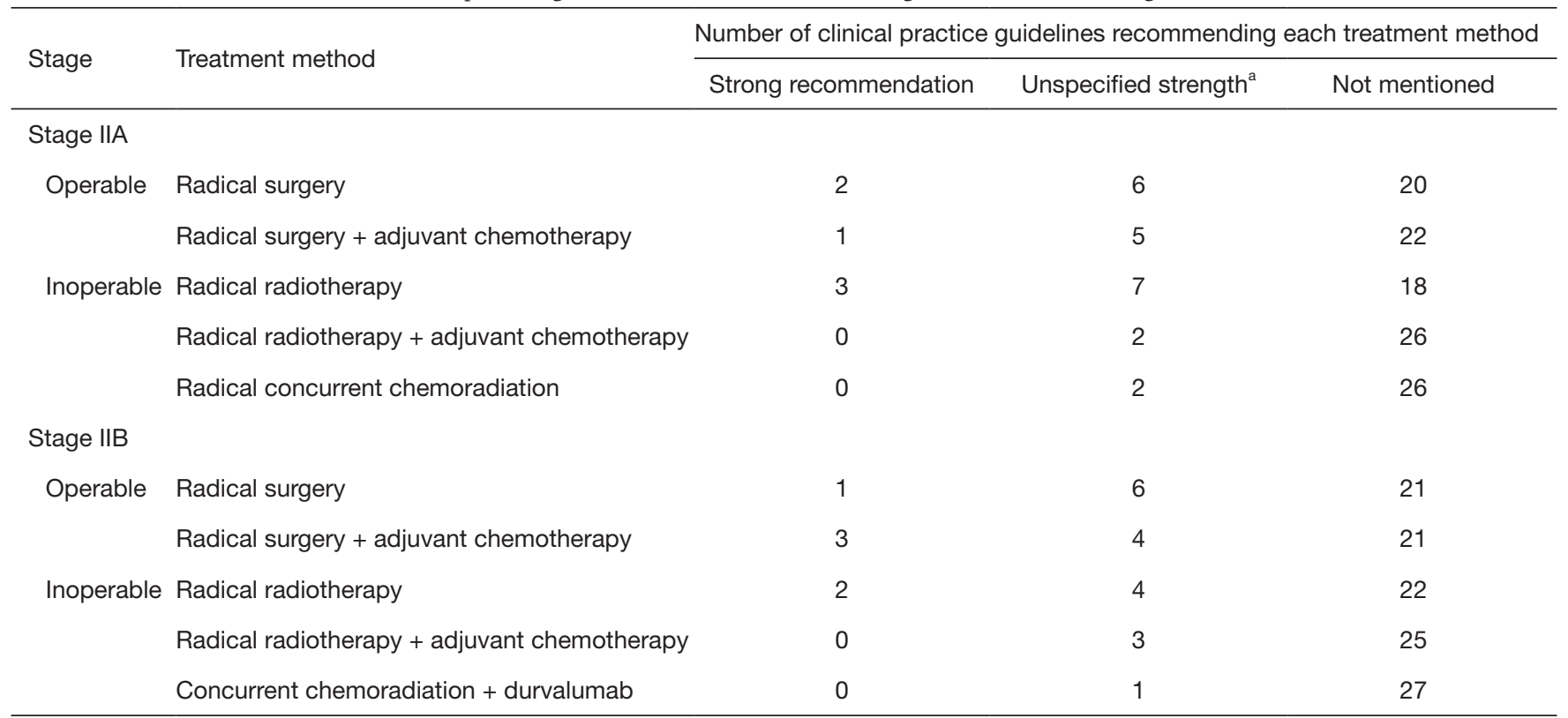

a, unspecified strength: the evidence was not graded in the guidelines, or the evidence was graded but the strength of the recommendation was not stated.

Table 4 Recommendations in the clinical practice guidelines for the treatment of stage III non-small cell lung cancer: guidelines dividing stage III to operable and inoperable only $(\mathrm{n}=14)$

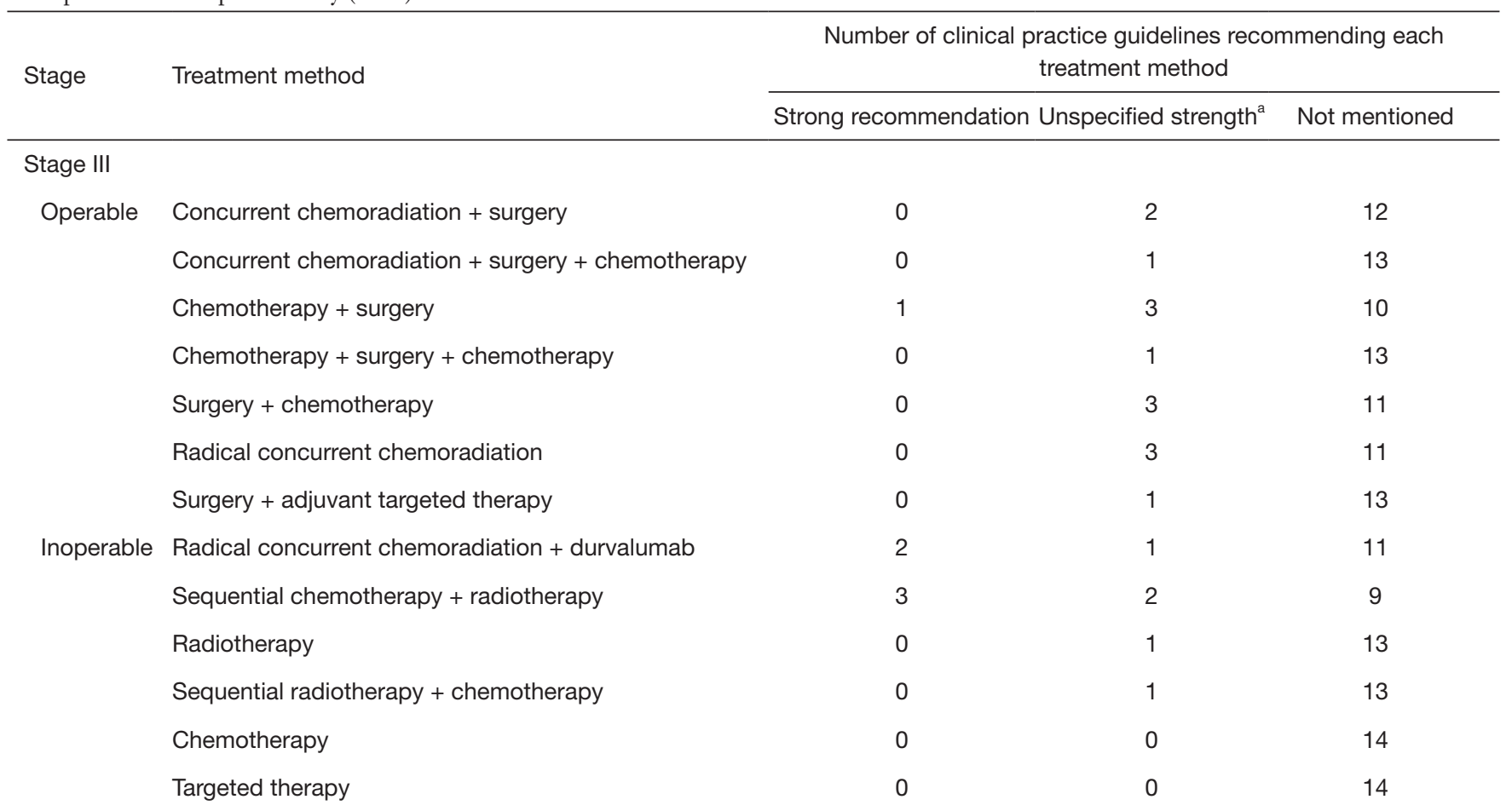

a , unspecified strength: the evidence was not graded in the guidelines, or the evidence was graded but the strength of the recommendation was not stated. 
Table 5 Recommendations in the clinical practice guidelines for the treatment of stage III non-small cell lung cancer: guidelines dividing stage III into three sub-stages IIIA, IIIB and IIIC $(n=14)$

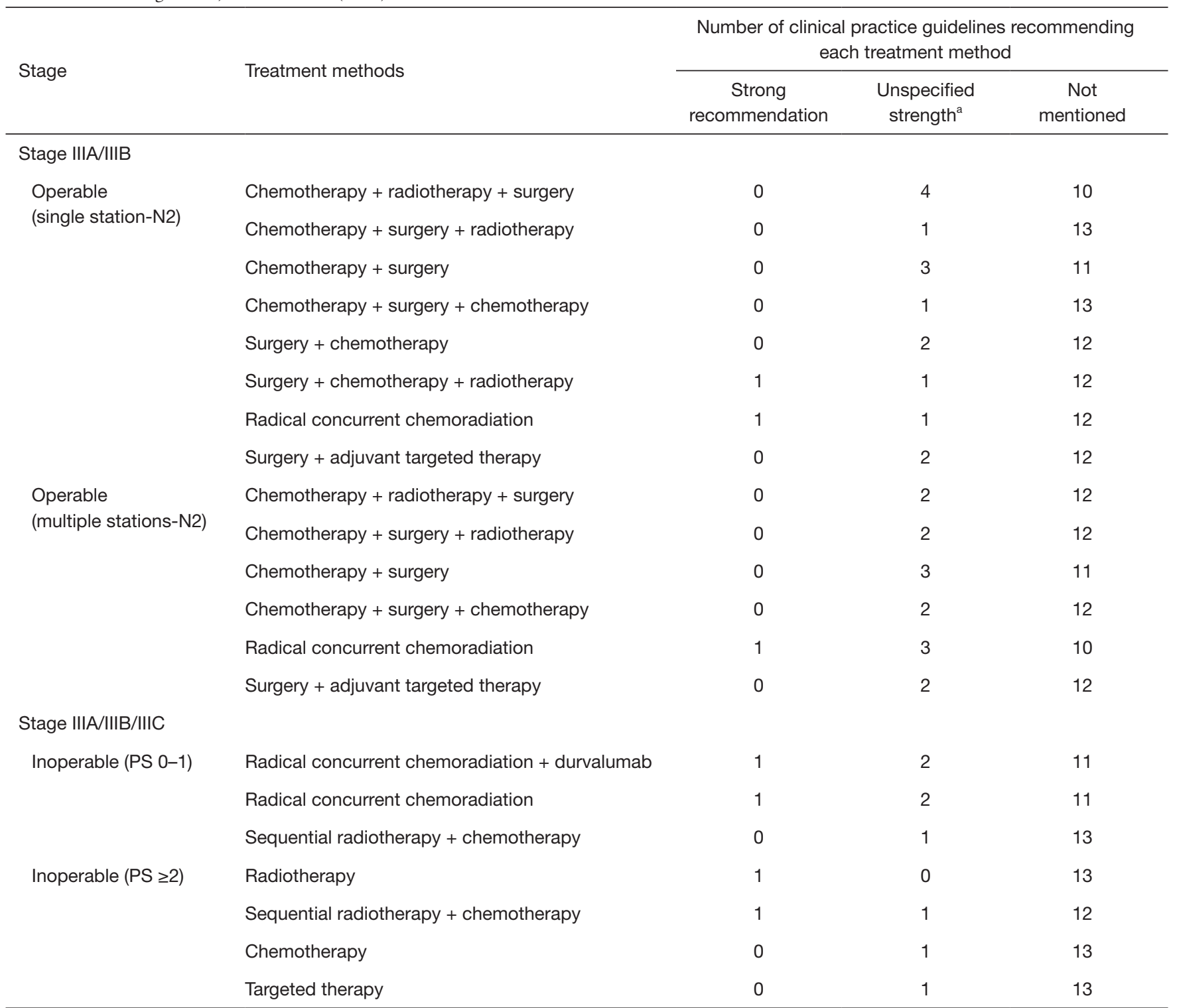

a , unspecified strength: the evidence was not graded in the guidelines, or the evidence was graded but the strength of the recommendation was not stated.

quality data. Nonetheless, definitive chemoradiation with durvalumab has shown improved progression-free and overall survival benefit as compared to placebo, and safety was similar between the groups (55). Therefore, some guidelines recommend radical concurrent chemoradiation together with durvalumab for patients with inoperable stage III NSCLC. That said, there is a major discrepancy in guidelines regarding inoperable stage III patients who have PDL $1<1 \%$, where little to no benefit was found in the
PACIFIC trial. These molecularly guided recommendations require further refinement and alignment at a global scale.

Accompanied with high level of evidence (56-58), recommendations on treatment of stage IV driver gene positive patients were consistent across the guidelines. For EGFR-mutation positive or ALK-rearrangement positive patients, targeted therapy is recommended as the primary choice for both first- and second-line treatment, followed by chemotherapy. Driver gene negative patients 
Table 6 Recommendations in the clinical practice guidelines for the treatment of stage IV non-small cell lung cancer

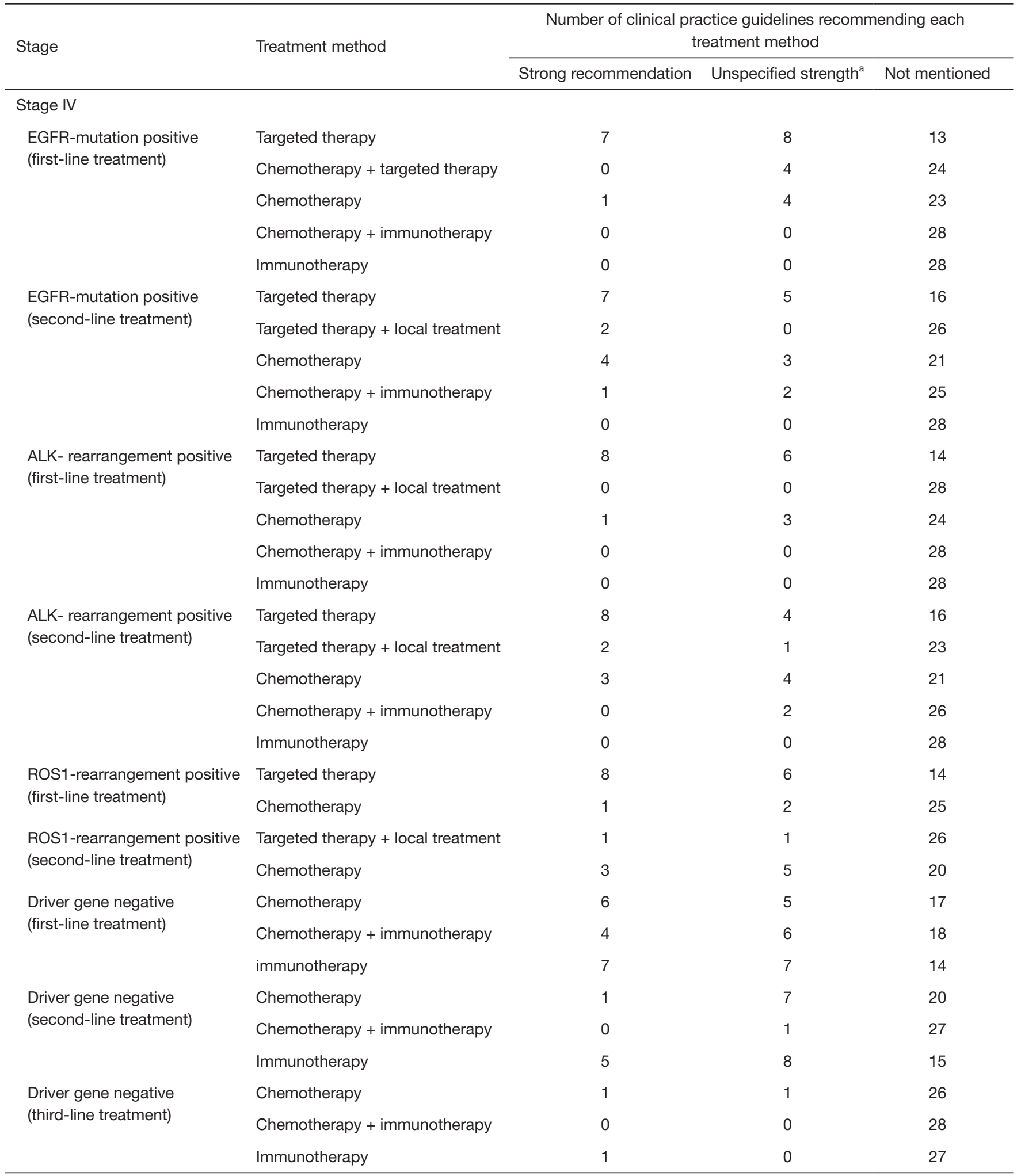

a , unspecified strength: the evidence was not graded in the guidelines, or the evidence was graded but the strength of the recommendation was not stated. 
account for $50-60 \%$ of patients with advanced NSCLC, and for these patients it is particularly important to adopt accurate evidence-based diagnosis methods and treatment strategies (59). Before the advent of immunotherapy, the standard treatment for Stage IV NSCLC was chemotherapy with platinum (60). The application of immunotherapy drugs in the treatment of NSCLC has completely changed the current treatment landscape (47-49). The results from the KEYNOTE-024 study on the five-year overall survival (OS) rate were very promising. The results showed that the fiveyear OS rate in the pembrolizumab single-agent treatment group was nearly twice as high as in the chemotherapy group (31.9\% vs. $16.3 \%)$. The median OS time was twice as long with pembrolizumab as with chemotherapy. These results show that immunotherapy significantly improves the survival of NSCLC patients (61-63). The recommendations for the first-line treatment of driver gene-negative nonsmall cells were consistent, which is likely due to the amount of high-quality evidence $(62,64)$. The choice between chemotherapy alone, chemotherapy with immunotherapy, or immunotherapy alone, should be made according to the expression status of PD-LI.

Inconsistency of recommendations across guidelines can result from multiple potential reasons. First, the time span when the guidelines were developed can cause heterogeneity, because the emergence of new evidence may overturn the old concepts and the change of the historical background can lead to a different interpretation of the same literature. It is therefore acceptable to see differences among guidelines according to publication year. Second, different countries and areas consider the local preferences when developing guidelines. Third, lack or poor quality of evidence may lead to the recommendations being put forward based on expert opinion rather than evidence. Fourth, different grading methods can form inconsistent recommendations based on the same evidence. Poor consistency across CPGs can confuse the guideline users, and we therefore advocate paying more attention to examining the controversies and updating guidelines timely to improve the consistency among CPGs.

The findings of our study can be considered important in several ways. For clinicians, especially oncologists, our research results can provide objective guidance for choosing the appropriate recommendations, but specialists in other fields can also benefit from these findings when assessing specialized guidelines. For medical researchers, our research presents the current status of NSCLC guidelines worldwide and give the opportunity to pay more attention to the existing gaps.

Our study had many strengths. We adopted a systematic review method to systematically search and analyze the NSCLC guidelines worldwide. We objectively reviewed the differences in recommendations for NSCLC diagnosis and treatment between the guidelines.

However, our study had also several limitations. First, we limited the search to CPGs published in Chinese or English. The lack of guidelines in other languages may affect the generalizability of our results. Second, the interpretation of recommendations is inherently subjective. However, we tried to alleviate this problem through building an interdisciplinary cooperation between specialists in different fields, including evidence-based medicine methodology. Third, we did not evaluate the details of the clinical content of the CPG recommendations, such as the surgical method and choice of drugs.

In summary, in the past three years, the development of CPGs for NSCLC has received extensive attention. The guidelines are updated quickly, but the quality of different guidelines varies, and some guidelines do not apply the essential tools of CPG development such as a grading system for the quality of evidence. Guidelines often lack consistency between recommendations, which may be affected by many factors. With the continuous revision and update of the guidelines, we are confident that future guidelines will be formulated with higher quality to form clear, definite and consistent recommendations for NSCLC diagnosis and treatment.

\section{Questions to be further discussed and considered}

\section{Question 1: What kind of subgroups do you think stage III should be divided into?}

\section{Expert opinion: Dr. Jonathan D. Spicer}

This is a very interesting question. Stage III is by far the most heterogenous stage in lung cancer with wide ranging biology at play. We have patients with T4 tumors and no nodal or distant metastasis and others with small primary disease and very bulky nodal progression. Certainly, the management of these very different anatomic scenarios is also quite different. Furthermore, as we better understand the molecular biology of curable stages of locally advanced lung cancer, driver mutation status and molecular markers like PD-L1 expression levels become increasingly relevant. The central purpose around staging is its ability to 
prognosticate survival outcomes. However, the primary clinical utility of staging is to assist with correct treatment assignments. Today, the correct treatment is hard to assign without molecular data. As an example, a stage III patient with operable disease who has an exon 19 EGFR mutation is more likely to be directed towards surgery and adjuvant chemo + TKI, while a patient with operable stage III disease may be offered a wide array of treatment options that include neoadjuvant therapy followed by surgery, surgery followed by adjuvant therapy or concurrent chemoradiation and immunotherapy! In this respect, the current staging system does not provide much help in terms of assigning the optimal treatment plan. If one believes that surgical resection is the optimal form of local control in stage III and is superior to concurrent radiation, perhaps categorizing stage III between operable and inoperable is the most rational way to proceed. Alternatively, if the focus is on the biology of the disease, a focus on driver mutation and molecular profile is most likely to predict outcome regardless of the treatment plan employed. Part of the challenge is establishing a categorization that is rational in design versus one that is dictated by existing and approved therapeutic options in play. For example, should a patient with inoperable stage IIIB lung adenocarcinoma and an ALK fusion be treated with adjuvant immunotherapy or adjuvant TKI therapy after concurrent chemoradiation? Most likely the survival of such a patient is quite different than that of one who does not have a targetable genetic alteration, just as the survival of patients with PD-L1 $<1 \%$ is very different from those with $>1 \%$ in the PACIFIC trial. Yet, phase 3 trials do not yet have results for all these scenarios. My bias would be to establish two primary categories: (I) classify by operability-in light of the poor PFS from PACIFIC, I feel that surgical resection continues to offer the best chance of cure when optimal systemic therapy is delivered; (II) classify by molecular profilePD-L1 status and presence of known targetable driver mutations would be most informative at this stage.

\section{Expert opinion: Mariano Provencio}

I think the first thing is to know if they are potentially resectable or not. A large group would be the N3, which would be unresectable from the start. Within those with N2 involvement, if it is multiple or single if it has bulky disease or not.

\section{Expert opinion: Noemi Reguart}

Resectable and non-resectable.

\section{Expert opinion: Tetsuya Mitsudomi}

(I) nonN2 IIIA group T3N1, T4N0/1;

(II) N2 IIIA group: (i) without extranodal invasion (a) incidental (cN0pN2), (b) single station (c) multiple stations, (ii) with extranodal invasion;

(III) N2 IIIB;

(IV) N3 (IIIB/IIIC).

\section{Expert opinion: Young Wha Koh}

Guidelines for the treatment of patients with stage III have not yet been established. Therefore, there has been a lot of controversy over stage III subgrouping. I think stage III should be divided into IIIa, IIIb, IIIc. There are significant differences in survival rates according to stage IIIa, IIIb, and IIIc. For a more precise treatment plan, systematic classification through the TNM stage is better.

\section{Question 2: Can immunotherapy be used in all driver gene negative patients? Do you think biomarker is necessary in choosing immunotherapy? Why?}

\section{Expert opinion: Dr. Jonathan D. Spicer}

I think it is clear at this point that the optimal biomarker of response to checkpoint inhibition is still lacking. PD$\mathrm{L} 1$ is helpful but not definitive. Recent results from the Checkmate 816 study seem to indicate that PD-L1 status does not really predict extent of response from chemoimmunotherapy. As such, it does seem unwise to claim at this stage that a biomarker level like tumor proportion score is required to assign a particular patient to an immunotherapy containing regimen. On the flip side, PDL1 TPS did seem to be very much associated with degree of efficacy of adjuvant durvalumab in the PACIFIC study, such that some jurisdictions did not approve the addition of durvalumab for patients after chemoradiation in PD-L1 $0 \%$ patients. At this point, I think data are too immature to mandate a biomarker as there seems to be efficacy in some cases even in the absence of high PD-L1. The addition of chemotherapy to immunotherapy seems to overcome some of the effects seen in low PD-L1 patients and this may be more relevant in curable stages of disease like stage III. My sense is that neoadjuvant studies looking at pathological response after various immunotherapeutic regimens are optimally poised to define the best biomarkers of biological response to immunotherapy and from these we may one day develop biomarkers that clearly outline which patients should not be offered immunotherapy. Until that point, I believe that patients who do not have detectable driver 
mutations should indeed be offered immunotherapy either alone or in combination with chemotherapy, radiation and surgery as the case may be.

\section{Expert opinion: Mariano Provencio}

Available clinical trial data establish different ranges of benefit depending on PD-L1 expression when immunotherapy is used alone but not in combination with chemotherapy. Even so, there is still a non-negligible proportion of patients with high expression who progress to treatment, so the markers known to date are still very imperfect.

\section{Expert opinion: Noemi Reguart}

Yes, if not contraindications and good PS conditions. PD-L1 Biomarker currently the best (albeit not optimal) biomarker for selection. Others TMB, MSI but not stablished in routine in lung cancer.

\section{Expert opinion: Tetsuya Mitsudomi}

For unresectable metastatic disease, Immunotherapy (with/without chemotherapy) is indicated in all driver gene mutation-negative patients if there are no factors that negate immunotherapy (such as ILD, uncontrolled autoimmune disease, post-transplantation, Earlier disease should be treated mainly by local therapies (surgery, radiation). PD-L1 TPS is important to determine whether to use chemotherapy in addition to IO drug. The role of TMB is still unclear.

\section{Expert opinion: Young Wha Koh}

I think that immunotherapy should be used in all driver gene negative patients. Many previous clinical trials have shown that immunotherapy improves survival and quality of life in driver gene negative patients. I think biomarkers are needed for immunotherapy selection. This is because immunotherapy is expensive and there is rare reaction such as hyperprogression after treatment.

\section{Question 3: How should clinicians make better treatment strategies for an individual patient when recommendations are inconsistent in CPGs?}

\section{Expert opinion: Dr. Jonathan D. Spicer}

This is a tough question. Obviously, clinicians want to follow practice guidelines that are developed using the best available evidence. However, the evidence does not always come at the same pace as one may need for an individual patient. As an example, we are currently lacking phase 3 data to support decisions for patients with inoperable stage 3 EGFR mutated lung cancer patients. This question highlights the discrepancy. Obviously, the preference would be to treat with chemoradiation and use adjuvant TKI in such a patient. But most jurisdictions will not have funding programs for such a treatment plan as it is not supported by phase 3 data addressing this specific scenario. Similarly, while there is phase 3 data to support chemo and TKI in a resected stage 3 patient, what do we do with a patient who was stage 3 at presentation by clinical and invasive mediastinal staging, received induction chemotherapy, now has ypT1N0 disease and is found to have and EGFR exon 19 mutation? Logically, this patient should be offered adjuvant TKI even though the final pathological stage is no longer stage 3 , the clinical course is likely to follow the same path as a patient who did not receive neoadjuvant chemotherapy. So, in summary, as we dissect the biology of lung cancer into increasingly precise sub-categories, we are likely to define subgroups for whom there are logical treatment combinations to which we should have access that are not necessarily supported by phase 3 trials. It is likely that CPGs over the coming years will remain woefully out of touch with the increasing complexity of what clinicians are facing in terms of decisions and in light of the rapidly growing data from which decisions are being made. This may not answer the question of how we can make better decisions, but certainly reveals that CPGs may not be the way. I believe that creating increased flexibility in terms of how we apply the evidence is very important and this should be accompanied by large-scale efforts for real-world data collection and prospective evaluation so as to periodically audit how this increased flexibility impacts care over time and can be curtailed when greater rigor needs to be applied or dedicated large scale phase 3 questions need to be answered.

\section{Expert opinion: Mariano Provencio}

Perhaps in the absence of clinical trials real-life data in specific situations may help. I believe that academic research should be strengthened to raise questions and solutions beyond interests linked to commercial approval.

\section{Expert opinion: Noemi Reguart}

This is a tricky question that seems to be drawn from the conclusions of your manuscript. I do strongly think this is not appropriate. Guidelines should give an objective level of evidence and recommendation. Whether if not we should 
carry out with guiltiness? otherwise if not convinced with local ones, others can be freely consulted.

\section{Expert opinion: Tetsuya Mitsudomi}

Areas where there is an inconsistency among different CPG are controversial area due to lack of evidence. Therefore, each different recommendation is not either good enough or bad enough. There will not be a big difference whichever CPG you take. In some cases, inconsistency may reflect the difference of social/economic/biologic characters of lung cancer in different geographic areas. Those include differences in drug approval, reimbursement, insurance, price of drugs, mutation frequency. Thus, if there is CPG intended to use in a particular country which is not in agreement with a global CPG, you should use the local CPGs.

\section{Expert opinion: Young Wha Koh}

The development of new drugs is very rapid, therefore a more recent CPG should be referred. If CPG has not yet been established, the latest information should be obtained from the Lung Cancer Society (IASLC World Conference on Lung Cancer or ASCO Annual Meeting).

\section{Acknowledgments}

The authors appreciate the academic support from the AME Reporting Guideline Collaborative Group.

Funding: This work was supported by Henan Province Health and Youth Subject Leader Training Project ([2020]60); Leading Talent Cultivation Project of Henan Health Science and Technology Innovation Talents (YXKC2020009); ZHONGYUAN QIANREN JIHUA (ZYQR201912118); Henan International Joint Laboratory of drug resistance and reversal of targeted therapy for lung cancer ([2021]10); Henan Medical Key Laboratory of Refractory lung cancer ([2020]27); Henan Refractory Lung Cancer Drug Treatment Engineering Technology Research Center ([2020]4); the 51282 project Leading Talent of Henan Provincial Health Science and Technology Innovation Talents ([2016]32); Huilan Charity Funda project (HL-HS2020-129).

\section{Footnote}

Reporting Checklist: The authors have completed the PRISMA reporting checklist. Available at http://dx.doi. org/10.21037/tlcr-21-423
Conflicts of Interest: All authors have completed the ICMJE uniform disclosure form (available at http://dx.doi. org/10.21037/tlcr-21-423). MP received grants from BMS and Roche, and consulting fees BMS, Astra Zeneca, MSD, Angem, Roche and Takeda; all outside the submitted work. TM received research grants from Boehringer Ingelheim, Asrtazeneca, Ono, Taiho, Daiichi-Sankyo and MSD; received payment or honoraria for lectures, presentations, speakers bureaus, manuscript writing or educational events from AstraZeneca, Novartis, Chugai, Amgen, MSD, EliLilly, Merck Biopharma, Pfizer and Boehringer Ingelheim; participated on a data safety monitoring board or advisory board of AstraZeneca, Novartis, Chugai, Amgen, MSD, Bristol Myers Squibb, Boehringer Ingelheim; and served as president of IASLC. JDS received research grants from AZ, Roche and Merck and honoraria and consulting fees from AZ, BMS, Merck, Protalix and CLS Therapeutics; outside the submitted work. The other authors have no conflicts of interest to declare.

Ethical Statement: The authors are accountable for all aspects of the work in ensuring that questions related to the accuracy or integrity of any part of the work are appropriately investigated and resolved.

Open Access Statement: This is an Open Access article distributed in accordance with the Creative Commons Attribution-NonCommercial-NoDerivs 4.0 International License (CC BY-NC-ND 4.0), which permits the noncommercial replication and distribution of the article with the strict proviso that no changes or edits are made and the original work is properly cited (including links to both the formal publication through the relevant DOI and the license). See: https://creativecommons.org/licenses/by-nc-nd/4.0/.

\section{References}

1. Wild CP, Weiderpass E, Stewart BW. World Cancer Report: Cancer Research for Cancer Prevention. Lyon, France: International Agency for Research on Cancer, 2020.

2. Siegel RL, Miller KD, Jemal A. Cancer statistics, 2020. CA Cancer J Clin 2020;70:7-30.

3. Nasim F, Sabath BF, Eapen GA. Lung Cancer. Med Clin North Am 2019;103:463-73.

4. Chen Z, Fillmore CM, Hammerman PS, et al. Non-smallcell lung cancers: a heterogeneous set of diseases. Nat Rev Cancer 2014;14:535-46. 
5. The L. Lung cancer: some progress, but still a lot more to do. Lancet 2019;394:1880.

6. Grimshaw JM, Russell IT. Effect of clinical guidelines on medical practice: a systematic review of rigorous evaluations. Lancet 1993;342:1317-22.

7. Buxton M. Implications of the appraisal function of the National Institute for Clinical Excellence (NICE). Value Health 2001;4:212-6.

8. Bironzo P, Di Maio M. A review of guidelines for lung cancer. J Thorac Dis 2018;10:S1556-63.

9. Kutob L, Schneider F. Lung Cancer Staging. Surg Pathol Clin 2020;13:57-71.

10. Akhurst T. Staging of Non-Small-Cell Lung Cancer. PET Clin 2018;13:1-10.

11. Tsao MS, Yoon JY. The eighth TNM classification for lung cancer-What is next? Lung Cancer 2018;121:97-8.

12. Feng SH, Yang ST. The new 8th TNM staging system of lung cancer and its potential imaging interpretation pitfalls and limitations with CT image demonstrations. Diagn Interv Radiol 2019;25:270-9.

13. Zhang XC, Lu S, Zhang L, et al. Guidelines for the diagnosis and treatment of anaplastic lymphoma kinasepositive and ROS1-positive non-small cell lung cancer in China. Zhonghua Bing Li Xue Za Zhi 2018;47:241-7.

14. Planchard D, Popat S, Kerr K, et al. Metastatic non-small cell lung cancer: ESMO Clinical Practice Guidelines for diagnosis, treatment and follow-up. Ann Oncol 2018;29:iv192-iv237.

15. Chinese guidelines for diagnosis and treatment of primary lung cancer 2018 (English version). Chin J Cancer Res 2019;31:1-28.

16. Nestle U, De Ruysscher D, Ricardi U, et al. ESTRO ACROP guidelines for target volume definition in the treatment of locally advanced non-small cell lung cancer. Radiother Oncol 2018;127:1-5.

17. Facchinetti F, Pilotto S, Metro G, et al. Treatment of metastatic non-small cell lung cancer: 2018 guidelines of the Italian Association of Medical Oncology (AIOM). Tumori 2019;105:3-14.

18. Majem M, Juan O, Insa A, et al. SEOM clinical guidelines for the treatment of non-small cell lung cancer (2018). Clin Transl Oncol 2019;21:3-17.

19. Wu YL, Planchard D, Lu S, et al. Pan-Asian adapted Clinical Practice Guidelines for the management of patients with metastatic non-small-cell lung cancer: a CSCO-ESMO initiative endorsed by JSMO, KSMO, MOS, SSO and TOS. Ann Oncol 2019;30:171-210.

20. Ponce S, Bruna J, Juan O, et al. Multidisciplinary expert opinion on the treatment consensus for patients with EGFR mutated NSCLC with brain metastases. Crit Rev Oncol Hematol 2019;138:190-206.

21. Schneider BJ, Ismaila N, Aerts J, et al. Lung Cancer Surveillance After Definitive Curative-Intent Therapy: ASCO Guideline. J Clin Oncol 2020;38:753-66.

22. Baldotto C, Gelatti A, Accioly A, et al. Lung Cancer and the COVID-19 pandemic: Recommendations from the Brazilian Thoracic Oncology Group. Clinics (Sao Paulo) 2020;75:e2060.

23. Akamatsu H, Ninomiya K, Kenmotsu H, et al. The Japanese Lung Cancer Society Guideline for non-small cell lung cancer, stage IV. Int J Clin Oncol 2019;24:731-70.

24. Berman AT, Jabbour SK, Vachani A, et al. Empiric Radiotherapy for Lung Cancer Collaborative Group multi-institutional evidence-based guidelines for the use of empiric stereotactic body radiation therapy for non-small cell lung cancer without pathologic confirmation. Transl Lung Cancer Res 2019;8:5-14.

25. De Marinis F, Barberis M, Barbieri V, et al. Diagnosis and first-line treatment of non-small cell lung cancer in the era of novel immunotherapy: recommendations for clinical practice. Expert Rev Respir Med 2019;13:217-28.

26. Passiglia F, Bertolaccini L, Del Re M, et al. Diagnosis and treatment of early and locally advanced non-smallcell lung cancer: The 2019 AIOM (Italian Association of Medical Oncology) clinical practice guidelines. Crit Rev Oncol Hematol 2020;148:102862.

27. Expert Panel on Thoracic Imaging:, de Groot PM, Chung JH, et al. ACR Appropriateness Criteria ${ }^{\circledR}$ Noninvasive Clinical Staging of Primary Lung Cancer. J Am Coll Radiol 2019;16:S184-95.

28. NICE guideline [NG122]: Lung cancer diagnosis and management. 2019. Available online: https://www.nice.org. uk/guidance/ng122

29. Clinical practice guideline for radiation therapy of nonsmall cell lung cancer (2020 version). Chin J Radiat Oncol 2020;29:599-607.

30. Passiglia F, Pilotto S, Facchinetti F, et al. Treatment of advanced non-small-cell lung cancer: The 2019 AIOM (Italian Association of Medical Oncology) clinical practice guidelines. Crit Rev Oncol Hematol 2020;146:102858.

31. Dingemans AC, Soo RA, Jazieh AR, et al. Treatment Guidance for Patients With Lung Cancer During the Coronavirus 2019 Pandemic. J Thorac Oncol 2020;15:1119-36.

32. Hanna NH, Schneider BJ, Temin S, et al. Therapy for Stage IV Non-Small-Cell Lung Cancer Without Driver 
Alterations: ASCO and $\mathrm{OH}(\mathrm{CCO})$ Joint Guideline

Update. J Clin Oncol 2020;38:1608-32.

33. Arrieta O, Cardona AF, Lara-Mejía L, et al. Recommendations for detection, prioritization, and treatment of thoracic oncology patients during the COVID-19 pandemic: the THOCOoP cooperative group. Crit Rev Oncol Hematol 2020;153:103033.

34. Park K, Vansteenkiste J, Lee KH, et al. Pan-Asian adapted ESMO Clinical Practice Guidelines for the management of patients with locally-advanced unresectable non-smallcell lung cancer: a KSMO-ESMO initiative endorsed by CSCO, ISMPO, JSMO, MOS, SSO and TOS. Ann Oncol 2020;31:191-201.

35. Travis WD, Dacic S, Wistuba I, et al. IASLC Multidisciplinary Recommendations for Pathologic Assessment of Lung Cancer Resection Specimens After Neoadjuvant Therapy. J Thorac Oncol 2020;15:709-40.

36. Non Small Cell Lung Cancer, Version 8.2020, NCCN Clinical Practice Guidelines in Oncology. 2020. Available online: https://www.nccn.org/guidelines/guidelinesdetail? category=1\&id=1450

37. Passaro A, Addeo A, Von Garnier C, et al. ESMO Management and treatment adapted recommendations in the COVID-19 era: Lung cancer. ESMO Open 2020;5:e000820.

38. Chinese Medical Association guidelines for clinical diagnosis and treatment of lung cancer (2019 edition). Zhonghua Zhong Liu Za Zhi 2020;42:257-87.

39. Guidelines of Chinese Society of Clinical Oncology (CSCO) Non-Small Cell Lung Cancer. 2020. Available online: http://meeting.csco.org.cn/MUser/CscoPeriodical/1/2?key word $=\&$ leibie $=\&$ pyear $=0 \&$ loc $=\mathrm{E} 9317455 \mathrm{E} 99 \mathrm{FF} 2 \mathrm{AE}$

40. Chinese Association for Clinical Oncologists. Clinical practice guideline for stage IV primary lung cancer in China (2020 version). Chin J Oncol 2020;42:1-16.

41. Mairinger T. Histology, cytology and molecular diagnostics of lung cancer. Pathologe 2019;40:649-61.

42. Brainard J, Farver C. The diagnosis of non-small cell lung cancer in the molecular era. Mod Pathol 2019;32:16-26.

43. Wu SG, Shih JY. Management of acquired resistance to EGFR TKI-targeted therapy in advanced non-small cell lung cancer. Mol Cancer 2018;17:38.

44. Singhi EK, Horn L, Sequist LV, et al. Advanced NonSmall Cell Lung Cancer: Sequencing Agents in the EGFR-Mutated/ALK-Rearranged Populations. Am Soc Clin Oncol Educ Book 2019;39:e187-97.

45. Patil T, Simons E, Mushtaq R, et al. Targeted therapies for ROS1-rearranged non-small cell lung cancer. Drugs Today
(Barc) 2019;55:641-52.

46. Arbour KC, Riely GJ. Systemic Therapy for Locally Advanced and Metastatic Non-Small Cell Lung Cancer: A Review. Jama 2019;322:764-74.

47. Yu H, Boyle TA, Zhou C, et al. PD-L1 Expression in Lung Cancer. J Thorac Oncol 2016;11:964-75.

48. Villanueva N, Bazhenova L. New strategies in immunotherapy for lung cancer: beyond PD-1/PD-L1. Ther Adv Respir Dis 2018;12:1753466618794133.

49. Tsoukalas N, Kiakou M, Tsapakidis K, et al. PD-1 and PD-L1 as immunotherapy targets and biomarkers in nonsmall cell lung cancer. J buon 2019;24:883-8.

50. Furlow B. PET-CT Cancer Imaging. Radiol Technol 2018;90:149CT-70CT.

51. Wang T, Wang Z. Application of Metabolic Parameters Measured by ${ }^{18} \mathrm{~F}-\mathrm{FDG}$ PET/CT in the Evaluation of the Prognosis of Non-small Cell Lung Cancer. Zhongguo Fei Ai Za Zhi 2019;22:167-72.

52. Doubeni CA, Wilkinson JM, Korsen N, et al. Lung Cancer Screening Guidelines Implementation in Primary Care: A Call to Action. Ann Fam Med 2020;18:196-201.

53. Tabchi S, Kassouf E, Rassy EE, et al. Management of stage III non-small cell lung cancer. Semin Oncol 2017;44:163-77.

54. Sata Y, Nakajima T, Yamamoto T, et al. Keys to successful induction chemoradiotherapy followed by surgery for stage III/N2 non-small cell lung cancer. Surg Today 2019;49:547-55.

55. Antonia SJ, Villegas A, Daniel D, et al. Overall Survival with Durvalumab after Chemoradiotherapy in Stage III NSCLC. N Engl J Med 2018;379:2342-50.

56. Ramalingam SS, Yang JC, Lee CK, et al. Osimertinib As First-Line Treatment of EGFR Mutation-Positive Advanced Non-Small-Cell Lung Cancer. J Clin Oncol 2018;36:841-9.

57. Khan M, Lin J, Liao G, et al. ALK Inhibitors in the Treatment of ALK Positive NSCLC. Front Oncol 2019;8:557.

58. Tran PN, Klempner SJ. Focus on Alectinib and Competitor Compounds for Second-Line Therapy in ALK-Rearranged NSCLC. Front Med (Lausanne) 2016;3:65.

59. Cui Y, Fang W, Li C, et al. Development and Validation of a Novel Signature to Predict Overall Survival in "Driver Gene-negative" Lung Adenocarcinoma (LUAD): Results of a Multicenter Study. Clin Cancer Res 2019;25:1546-56.

60. Manegold C. Chemotherapy for advanced non-small cell lung cancer: standards. Lung Cancer 2001;34 Suppl 
2:S165-70.

61. Reck M, Rodríguez-Abreu D, Robinson AG, et al. Updated Analysis of KEYNOTE-024: Pembrolizumab Versus Platinum-Based Chemotherapy for Advanced NonSmall-Cell Lung Cancer With PD-L1 Tumor Proportion Score of 50\% or Greater. J Clin Oncol 2019;37:537-46.

62. Nosaki K, Saka H, Hosomi Y, et al. Safety and efficacy of pembrolizumab monotherapy in elderly patients with PDL1-positive advanced non-small-cell lung cancer: Pooled

Cite this article as: Zhang $\mathrm{Z}$, Yang S, Ma Y, Zhou H, Wu X, Han J, Hou J, Hao L, Spicer JD, Koh YW, Provencio M, Reguart N, Mitsudomi T, Wang Q. Consistency of recommendations for the diagnosis and treatment of non-small cell lung cancer: a systematic review. Transl Lung Cancer Res 2021;10(6):2715-2732. doi: 10.21037/tlcr-21-423 analysis from the KEYNOTE-010, KEYNOTE-024, and KEYNOTE-042 studies. Lung Cancer 2019;135:188-95.

63. Nasser NJ, Gorenberg M, Agbarya A. First line Immunotherapy for Non-Small Cell Lung Cancer. Pharmaceuticals (Basel) 2020;13:373.

64. Palumbo G, Carillio G, Manzo A, et al. Pembrolizumab in lung cancer: current evidence and future perspectives. Future Oncol 2019;15:3327-36. 


\section{Search Strategy}

\section{PubMed (n=414)}

\#1. Lung Neoplasms [MeSH]

\#2. lung neoplasm*[Title/Abstract]

\#3. lung cancer[Title/Abstract]

\#4. lung[Title]

\#5. OR \#1-\#4

\#6. "Guideline"[Publication Type]

\#7. $\quad$ "Practice Guideline"[Publication Type]

\#8. "guideline*"[Title]

\#9. "guidance ${ }^{*}[$ Title]

\#10. "recommendation*"[Title]

\#11. OR \#6-\#10

\#12. \#5 AND \#11

\#13. Lim2018/1/1-present

\section{CBM (n=150)}

\#1“肺肿瘤”[不加权 : 扩展 ]

\#2 “指南”[ 不加权 : 扩展 ]

\#3 “肺癌” [ 常用字段 : 智能 ] OR” 肺肿瘤 “[ 常用字段 : 智能 ]

\#4 ”指南 “[ 常用字段 : 智能 ] OR” 推荐意见 “[ 常用字段 : 智能]

\#5 \#1 OR \#3

\#6 \#2) OR \#4

\#7 \#5 AND \#6

\#8 \#7 AND 2018-2020

\section{Wanfang ( $=54)$}

\#1 主题 : 肺癌

\#2 主题 : 肺肿瘤

\#3 \#1 OR \#2

\#4 题名: 指南

\#5 题名 : 推荐意见

\#6 \#4 OR \#5

\#7 \#3 AND \#6

\#8 \#7 AND 2018-2020

\section{CNKI (n=33)}

\#1 主题:(肺癌 or 肺肿瘤) and 题名:( 指南 or 推荐意见 )

\#2 \#1 AND 2018-2020

\section{WHO (n=0)}

\#1 lung cancer

\#2 $\lim 2018-2020$

NICE $(n=1)$

\#1 Lung cancer OR \#2 Lung Neoplasms

\#3 $\lim 2018-2020$ 
GIN (n=4)

\#1 Lung cancer

\#2 $\lim 2018-2020$

\section{SIGN $(n=0)$}

\#1 guideline

\#2 Lung cancer

\#3 \#1 AND \#2

\#4 \# $\lim 2018-2020$

\section{$\operatorname{NCCN~(n=1)~}$}

\#1 Non-small Cell Lung Cancer AND 2018-2020 